\title{
Boundary-Specific Cost Functions for Quantitative Airway Analysis
}

\author{
Atilla P. Kiraly ${ }^{1}$, Benjamin L. Odry ${ }^{1}$, David P. Naidich ${ }^{2}$, \\ and Carol L. Novak ${ }^{1}$ \\ ${ }^{1}$ Siemens Corporate Research, Princeton, NJ \\ \{atilla.kiraly, benjamin.odry, carol.novak\} @siemens.com \\ ${ }^{2}$ Department of Radiology, NYU Medical University, New York, NY \\ david.naidich@nyume.org
}

\begin{abstract}
Computed tomography (CT) images of the lungs provide high resolution views of the airways. Quantitative measurements such as lumen diameter and wall thickness help diagnose and localize airway diseases, assist in surgical planning, and determine progress of treatment. Automated quantitative analysis of such images is needed due to the number of airways per patient. We present an approach involving dynamic programming coupled with boundary-specific cost functions that is capable of differentiating inner and outer borders. The method allows for precise delineation of the inner lumen and outer wall. The results are demonstrated on synthetic data, evaluated on human datasets compared to human operators, and verified on phantom CT scans to sub-voxel accuracy.
\end{abstract}

\section{Introduction}

Diseases and abnormalities of the airways can manifest themselves as observable changes in airway lumen diameters and wall thicknesses [1]. These changes can also occur in response to treatment. High-resolution computed tomography (CT) images of the chest offer high resolution views of the airways that potentially allow one to obtain detailed quantitative measurements. However systematic analysis of such images is not feasible without automation due to the sheer number of airways involved. Certain airway abnormalities can affect only localized regions, making analysis of multiple locations throughout the lungs necessary. Automated analysis of such images helps reduce or eliminate reader variability and allows for the entire patient dataset to be analyzed within a reasonable amount of time.

Automated methods for quantitative airway analysis attempt to precisely determine the boundaries of the inner lumen and outer airway wall. These methods face several challenges in providing accurate measurements, especially in smaller airways. Partial volume artifacts result when regions of different densities exist within the volume of a single voxel [2], making it more difficult to find the exact wall location. Additional difficulties arise from the scanner's Point Spread Function (PSF) [3] and CT reconstruction artifacts, which distort the image and create additional noise. Finally, a problem specific to the airways is identifying the outer wall boundary when there is an adjacent artery, which in many situations, obscures the true boundary. 
The full-width half-max (FWHM) approach is a popular method that is frequently used as a point of comparison. This method determines the location of the inner and outer boundaries based on the maximum intensity within the wall and the minimum intensities of the air region [4]. The location of the intensity computed from the average of these two is judged as the wall position. This method has been shown to underestimate the inner lumen boundary and overestimate the outer boundary [3]. In [5] the airway segmentation added robustness by defining an initial boundary.

A sub-voxel accurate method for determining airway lumen and wall thickness was presented in $[3,6]$. These methods estimate the scanner's PSF to help fit a cylindrical model to the data. Although the results show high accuracy, the method depends on an assumed cylindrical shape, which is not always the case for the airways.

In [7], a phase-congruency space is used to recover the borders by predicting intensity crossing points obtained from different reconstruction kernels. The authors suggest it as a "bronze standard" since it shows little variance with different reconstruction kernels and is accurate up to discretization error.

A group of methods depend on an optimal fit based upon gradient computations. In [12], a dual tube model is fit to the inner and outer lumina. Fully automated methods often depend on a definition of the airway centerlines [8-11] to offer a central location as well as a perpendicular direction to perform the measurement. The above methods also have these requirements. An alternate method eliminates the need for direction vectors by using a sphere [13]. Although subvoxel accuracy is achieved, only the average diameter is computed. A promising method [14] finds the borders directly from the airway segmentation, allowing for an inner lumen definition without a dependence on airway centerlines. However, the method's accuracy is not yet established.

Minimum-path based methods operate by defining a minimum cost path through a cost function image via dynamic programming. This concept was originally applied to vessels $[15,16]$, but was adapted for airways $[11,17]$. In these applications, the data is resampled into a polar space and two balanced edge filters are used to obtain an accurate inner lumen boundary. The results show excellent accuracy and robustness, but the defined cost function limits its potential for obtaining the outer boundary and can make it vulnerable to other strong edge artifacts.

We propose a dynamic programming approach allowing for accurate inner and outer wall measurements. Our paper introduces cost functions targeted specifically for the inner and outer boundaries of airway walls. The functions can also be individually tuned to particular sizes, and increase robustness by limiting artifacts from nonboundary edges. Additionally, the determined inner border is used to influence the computation of the outer border to prevent intersection.

\section{Method}

The method computes cost functions locally at each site perpendicular to the direction of the airway. The airway centerlines are used to obtain these sites and directions. The cost functions are based on an airway model of a very low density lumen, a high density airway wall, and an intermediate density parenchyma outside the wall. 


\subsection{Airway Centerline Extraction}

The airway centerlines are the prerequisite for fully automated analysis. They describe airway tree hierarchy as a series of branches where each branch contains a series of sites giving the lumen center location as well as a direction heading. This location and the perpendicular plane defined by the heading give the two necessary components to define an isotropic 2D cross-sectional image of the site, allowing measurements to be computed at that point. Fig 1a shows a 2D image obtained from a given location and direction. We used a two step process of skeletonization of the segmented airways followed by refinement to obtain smooth centerlines. Complete details and validation are found in [10,5]. Other centerline methods can be used for this step [8-13]. Sites can also be manually specified if a single airway location is to be measured.

\subsection{Boundary Determination}

Given the 2D cross-sectional image of the site to be measured, it is first radially resampled into a polar form, as shown in Fig. 1b. The vertical direction corresponds to the distance from the airway center, while the horizontal direction corresponds to different angles around the airway axis. We used 360 different angles to form the images. Note that in Figs. 1b through 1e the images are horizontally compressed for space and are sampled to a sub-pixel level.

This polar image $\mathrm{C}_{\text {polar }}$ is used to create two cost function images, $\mathrm{C}_{\text {inner }}$ and $\mathrm{C}_{\text {outer }}$, (Figs. 1d and 1e) corresponding to the inner and outer boundaries. These cost functions assign a cost to each pixel in the radial image. The goal is to find a path with minimum cost spanning the horizontal length of the cost function image. A dynamic programming method is applied with the constraint that the vertical level of the starting position corresponds to that of the ending position [18]. Additionally, the path must be piece-wise congruent.

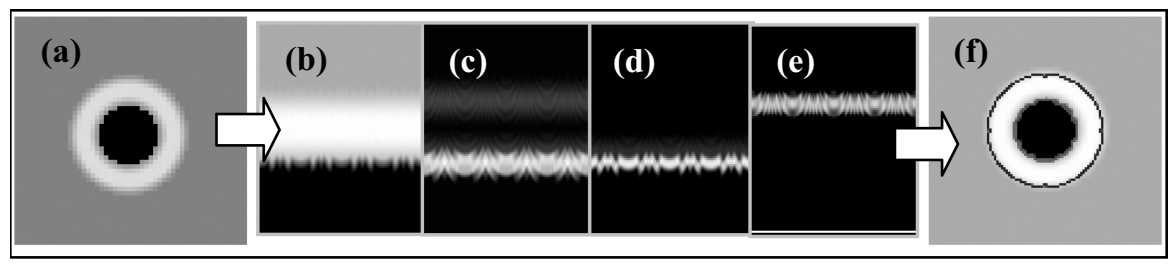

Fig. 1. Outline of the proposed method on synthetic airway data. The perpendicular cross section of the airway (a) is radially resampled (b). Cost functions are then determined from resampled image. (Brighter response $=$ lower cost) $(\mathrm{c})$ is a previously proposed cost function [11]. The proposed cost functions (d) and (e) are targeted towards the inner and outer boundaries. Horizontal paths through the cost functions then determine the boundaries as shown in (f).

Since two boundaries are determined, two minimum paths are computed. In the proposed method, first the inner boundary is determined. This boundary is then used to assign very high cost to the inner portions of the outer boundary cost function to 
prevent the two boundaries from crossing. The computed outer boundary then never intersects the inner boundary. The radially transformed image creates an almost linear structure from the airway wall along with a dark-light-dark pattern in the vertical direction, as illustrated in Fig. 2 (top). A straighter airway wall can also be induced by incrementally offsetting the image with regards to the segmentation of the airway lumen [11]. We make use of the unique placement of the wall within the polar image to accurately determine both boundaries, as shown in Fig. 2.

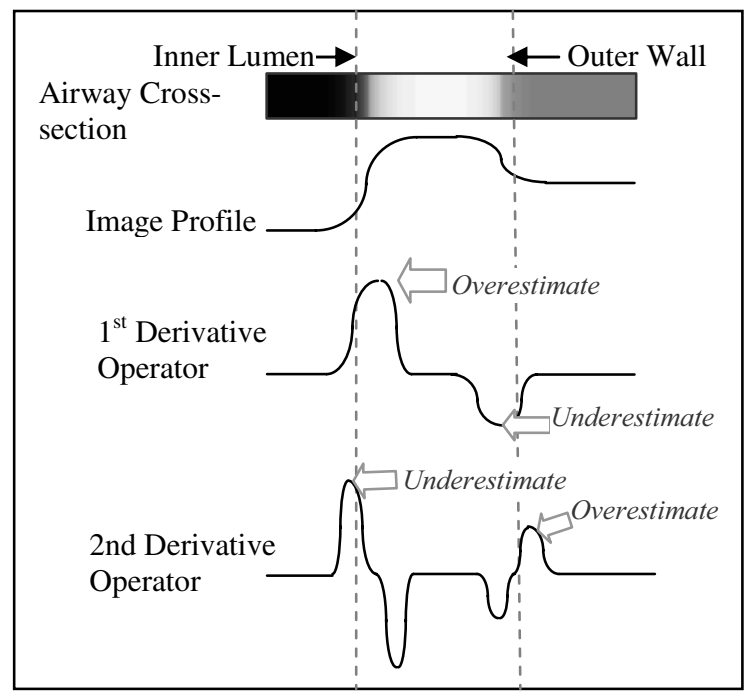

Fig. 2. An illustration of two directional filters that are combined to accurately detect inner and outer airway boundaries. A cross-section of an airway along a radial line is shown on top. The result of the 1st and 2nd order derivative operators are shown below. Examining the extremum, the 1 st derivative operator overestimates the inner lumen while underestimating the outer diameter. The opposite is true for the positive extremum in the 2 nd derivative operator. Hence, combining variations of each of these operators can allow for accurate localization of the inner lumen or outer wall.

We propose the following cost functions to allow for accurate inner and outer boundary characterization:

$$
\begin{aligned}
& C_{\text {inner }}=w_{i} \cdot H\left(D_{y}^{\prime}\left(C_{\text {polar }}\right)\right) \cdot D_{y}^{\prime}\left(C_{\text {polar }}\right)+\left(1-w_{i}\right) \cdot D_{y}^{\prime \prime}\left(C_{\text {polar }}\right), \text { and } \\
& C_{\text {outer }}=w_{o} \cdot\left(1-H\left(D_{y}^{\prime}\left(C_{\text {polar }}\right)\right) \cdot D_{y}^{\prime}\left(C_{\text {polar }}\right)+\left(1-w_{o}\right) \cdot D_{y}^{\prime \prime}\left(C_{\text {polar }}\right),\right.
\end{aligned}
$$

where $\mathrm{H}()$ is the Heaviside step function, returning 1 for positive values and 0 for negative values. These functions are composed of linear combinations of small scale directional $1^{\text {st }}$ and $2^{\text {nd }}$ order derivatives of the image $\mathrm{C}_{\text {polar }}$. The terms $\mathrm{w}_{\mathrm{i}}$ and $\mathrm{w}_{\mathrm{o}}$ have a possible range of $[0,1]$ and determine the weightings applied to each directional derivative to balance the under and over estimations. The inner boundary filter (1) is 
composed of a linear combination of the positive portions of the 1st derivative and the 2nd order derivative. As shown in Fig. 2, the 1st derivative operator overestimates while the 2 nd derivative operator underestimates. The outer border filter consists of a linear combination of the inverted negative portion of the 1 st derivative and the 2 nd order derivative. In this case, the 1st order derivative underestimates while the 2nd order derivative overestimates.

The results of these filters on synthetic data are shown in Fig. 1d and 1e. The outer boundary produces a weaker response relative to the inner boundary as predicted by Fig. 2. Note that this weaker response has no impact on the proposed method since the boundaries are computed separately. Also note that in each case the particular boundary not targeted has virtually no response.

The cost function of a previously proposed method [11] does not differentiate between inner and outer walls. Both boundaries appear in the cost function, as shown in Fig. 1c. This effect can distract the inner boundary computation. Further distractions can result in an emphasis of non-linear edges. This latter effect was compensated for by giving emphasis to specific edges depending upon gradient direction. This step can also be applied to the proposed method, but the focus is on developing specialized filters.

Fig. 3 shows an example of derived cost functions from a real airway along with the resultant boundaries. The adjacent artery creates an error in the outer boundary when using the standard minimum path algorithm, as shown in the left image. On the right, using the exact same cost functions, the minimum path is computed by assigning an additional cost for deviating from the predicted straight border. Note that imposing this additional cost does not significantly impact the inner boundary determined. Additionally possibilities are discussed in the conclusion.

Since a linear combination of underestimates and overestimates is involved, their weighting must be calibrated to produce accurate border estimates across different size ranges. These calibration parameters are obtained from materials of similar density to airways to account for the PSF and partial volume effects at different scales. During measurement, the airway segmentation is used to determine an inner size estimate and a linear interpolation between calibrated parameters of the nearest scale is applied for both parameters. It is possible to incorporate an outer size estimate to determine $\mathrm{w}_{\mathrm{o}}$ separately.

\section{Results}

Two different evaluations were performed. The method was first calibrated and evaluated on a scanned phantom airway. The phantom consists of 5 tubes of known dimensions, varying in inner diameter from $2 \mathrm{~mm}$ to $10 \mathrm{~mm}$ with wall thicknesses from $1 \mathrm{~mm}$ to $3 \mathrm{~mm}$. It is constructed of PVC pipe and nylon tubing and scanned at a voxel resolution of $0.35 \times 0.35 \times 0.5 \mathrm{~mm}^{3}$. We determined values of $\mathrm{w}_{\mathrm{i}}=[0.17,0.22,0.15,0.30$, $0.27]$ and $\mathrm{w}_{\mathrm{o}}=[0.49,0.17,0.32,0.20,0.21]$ for the range of sizes to provide optimal measurements and then applied them along multiple locations of the same tube. Again, linear interpolation is used based upon the size estimate from the segmentation. The method accurately measured the tube dimensions, demonstrating the 
validity of the cost function in determining the true boundaries to sub-voxel accuracy. Table 1 lists the errors and standard deviations.

The second experiment compared the measurements against two operators on a human airway CT dataset with $0.58 \times 0.58 \times 0.79 \mathrm{~mm}^{3}$ voxel resolution. A total of 34 airways with lumen diameters ranging from 1.2 to $3.6 \mathrm{~mm}$ and outer diameters ranging from 3.2 to $6.1 \mathrm{~mm}$ were randomly selected. Each operator independently performed inner and outer diameter measurements twice using digital calipers. Some locations included nearby arteries. The results show that the method produces measurement results within the error of the human operators. In the smallest airway, the method produced the largest variation $(0.5 \mathrm{~mm})$, although still achieving sub-voxel accuracy.

The average running time for each site was $1.1 \mathrm{~s}$. The path computation occupied the majority of the time $(70 \%)$ at $390 \mathrm{~ms}$ per path on average.

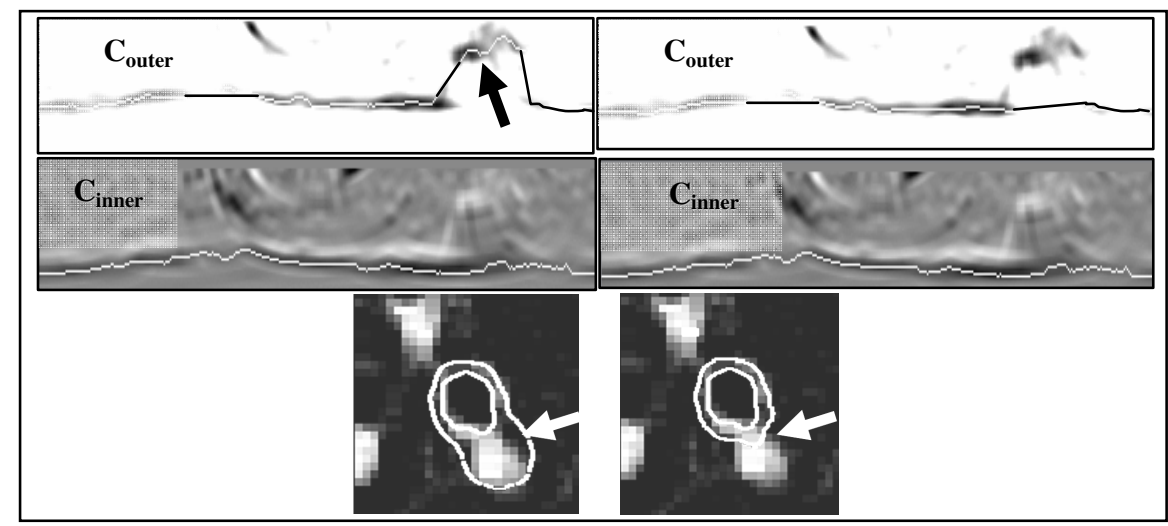

Fig. 3. The left and right sides show a comparison of two different minimum path computation methods. In both cases the results for the outer wall filter (top) and the inner wall filter (middle) are shown along with the minimum cost path. In each case the cost functions are the same with lower gray levels indicating lower cost. The subsequent boundary results are shown at the bottom. In the left case, a standard minimum path was computed, resulting in an error with the outer boundary due to the artery, (white arrow). In the right case, the path cost was increased when not heading straight. The outer boundary is correctly captured. Note that the inner boundary has not changed with this additional requirement.

Table 1. Absolute errors and standard deviations for the computed inner and outer diameters obtained from phantom data of hollow cylinders of known radii and wall thickness.

\begin{tabular}{ccccc}
\hline & $\begin{array}{c}\text { Mean Absolute } \\
\text { Error of Inner } \\
\text { Diameter }(\mathrm{mm})\end{array}$ & $\begin{array}{c}\text { Mean Absolute } \\
\text { Error of Outer } \\
\text { Diameter }(\mathrm{mm})\end{array}$ & $\begin{array}{c}\text { Std. Dev. Inner } \\
\text { Diameter }(\mathrm{mm})\end{array}$ & $\begin{array}{c}\text { Std. Dev Outer } \\
\text { Diameter }(\mathrm{mm})\end{array}$ \\
\hline Tube 1 & 0.02 & 0.03 & 0.0215 & 0.0259 \\
Tube 2 & 0.05 & 0.06 & 0.0417 & 0.0661 \\
Tube 3 & 0.11 & 0.07 & 0.0766 & 0.0772 \\
Tube 4 & 0.06 & 0.30 & 0.0551 & 0.0408 \\
Tube 5 & 0.15 & 0.10 & 0.1014 & 0.0549 \\
\hline
\end{tabular}




\section{Conclusions}

We have presented a method based on boundary-specific cost functions for quantitative airway measurements. Previous airway cost function methods use generalized cost functions not geared toward specific boundaries and produce extraneous responses. The proposed functions accurately target specific boundaries while producing little or no response to other boundaries. The computed inner path was also used to influence the outer cost function. This feature prevented the outer boundary from intersecting the inner boundary.

The method demonstrated robustness in cases of image noise and adjacent arteries when enforcing a straight path. Further experiments in the cost function definition and minimum path computation can be done to determine still more robust methods. Additional possibilities include allowing both paths to influence each other during computation as opposed to sequential computation. Modifications to the cost functions seem promising. Applying the Heaviside function to the second derivative operator eliminates negative responses, which may increase robustness. We find that assigning higher cost to regions above the high intensity areas occupied by arteries eliminates the need for enforcing a straighter path in cases such as Fig. 3. This change defines the cost functions with elements taken directly from the polar image in addition to the operators. Calibration based upon wall thickness also seems promising.

The method demonstrated accurate inner lumen and outer wall boundary determination for airway walls within phantom and human airway data. Further tests on multiple phantoms and reconstruction kernels are the subject of future work. Currently, it appears that the method needs re-calibration for different reconstruction kernels and CT-scanner machines. Finally, measurement tests with more human operators and locations will help further establish the method's robustness.

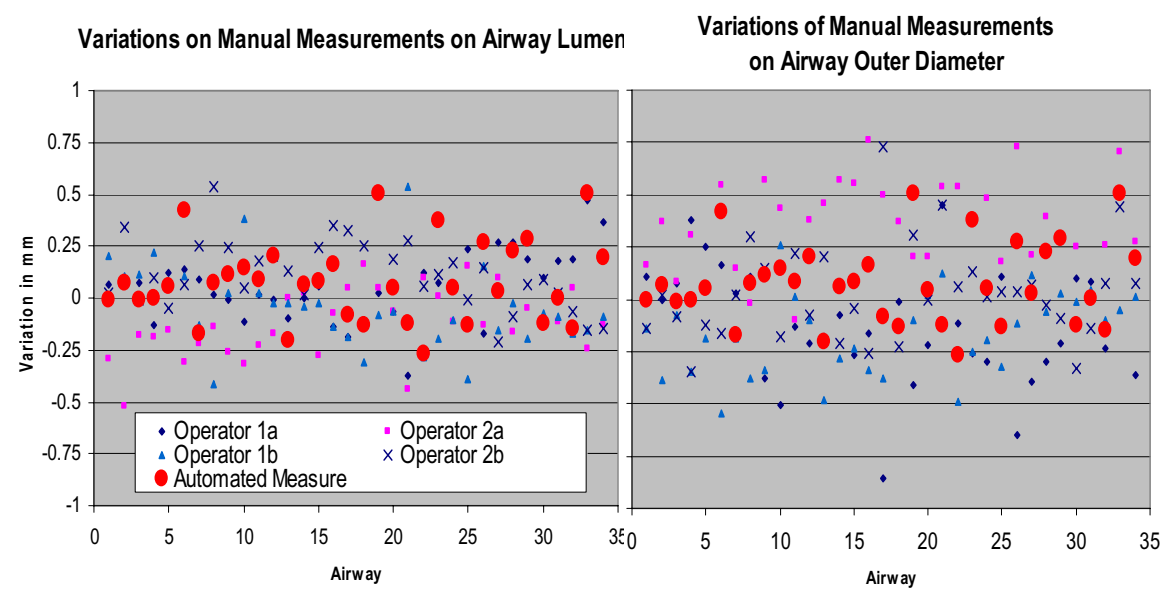

Fig. 4. A comparison of the proposed method against two human operators on a total of 34 airways. Operator $1 \mathrm{a}$ and $1 \mathrm{~b}$ refer to the measurements of the first operator at times 1 and 2 . Similarly $2 \mathrm{a}$ and $2 \mathrm{~b}$ correspond to operator 2 's measurements at times 1 and 2 . The results show that the obtained measurements are within the range of the inter- and intra- operator measurement variation. No airway size dependent variation was noticed. 


\section{References}

1. Matsuoka, S., Kurihara, Y., Nakajima, Y., Niimi, H., Ashida, H., Kaneoya, K.: Serial Change in Airway Lumen and Wall Thickness at Thin-Section CT in Asymptomatic Subjects. Radiology (December 2004)

2. Santago, P., Gage, H.D.: Statistical Models of Partial Volume Effect. IEEE Trans. Img. Proc. pp. 1531-1540 (November 1995)

3. Reinhardt, J.M., D'Souza, N.D., Hoffman, E.A.: Accurate measurement of intra-thoracic airways. IEEE Trans Med. Imag. 16(6), 820-827 (1997)

4. Nakano, Y., Muro, S., Sakai, H., Iría, T., Chin, K., Tsukino, M., Nishimura, K., Itoh, H., Pare, P.D., Hogg, J.C., Mishima, M.: Computed Tomographic Measurements of Airway Dimensions and Emphysema in Smokers. J. Respiratory Crit. Care Med. 161, 574-580 (2000)

5. Kiraly, A.P., Reinhardt, J.M., Hoffman, E.A., et al.: Virtual bronchoscopy for quantitative airway analysis. SPIE Medical Imaging 20055746 (2005)

6. Saba, O., Hoffmann, E.A., Reinhardt, J.M.: Maximizing quantitative accuracy of the lung airway lumen and wall measures obtained from X-ray CT imaging. J. Applied Physiology 95, 1063-1095 (2003)

7. Estépar, R.S.J., Washko, G.G., Silverman, E.K., Reilly, J.J., Kikinis, R., Westin, C.F.: Accurate Airway Wall Estimation Using Phase Congruency. In: Larsen, R., Nielsen, M., Sporring, J. (eds.) MICCAI 2006. LNCS, vol. 4191, Springer, Heidelberg (2006)

8. Preteux, F., Fetita, C.I., Grenier, P., Capderou, A.: Modeling, segmentation, and caliber estimation of bronchi in high-resolution computerized tomography. J. Electron. Imag. 8(1), 36-45 (1999)

9. Mori, K., Suenaga, Y., Toriwaki, J.: Automated anatomical labeling of the bronchial branch and its applications to the virtual bronchoscopy. Med. Imag. 19(2), 103-114 (2000)

10. Kiraly, A.P., Helferty, J.P, Hoffman, E.A., McLennan, G., Higgins, W.E.: 3D Path Planning for Virtual Bronchoscopy. IEEE Trans. Med. Imag. 23(11) (2004)

11. Tschirren, J., Hoffman, E.A., McLennan, G., Sonka, M.: Intrathoracic Airway Trees: Segmentation and Airway Morphology Analysis From Low-Dose CT Scans. IEEE Trans. Med. Imag. 24(12) (2005)

12. Odry, B.L., Kiraly, A.P., Novak, C.L., et al.: Automated airway evaluation system for multi-slice computed tomography using airway lumen diameter, airway wall thickness and broncho-arterial ratio. In: Proc. SPIE Medical Imaging 2006, vol. 6143 (2006)

13. Wiemker, R., Blaffert, T., Bülow, T., Renisch, S., Lorenz, C.: Automated assessment of bronchial lumen, wall thickness and bronchoarterial diameter ratio of the tracheobronchial tree using high-resolution CT. I.C.S. 1268 CARS (2004), pp. 973-977 (2004)

14. Saragalia, A., Fetita, C.I., Preteux, F.J.: Airway wall thickness assessment: a new functionality in virtual bronchoscopy investigation. SPIE Medical Imaging (2007)

15. Fleagle, S.R., Johnson, M.R., Wilbricht, C.J., Skorton, D.J., Wilson, R.F., White, C.W., Marcus, M.L., Collins, S.M.: Automated Analysis of Coronary Arterial Morphology in Cineangiograms: Geometric and Physiologic Validation in Humans. IEEE Trans. Med. Imag.8(4) (1989)

16. Sonka, M., Reddy, G.K., Winniford, M.D., Collins, S.M.: Adaptive Approach to Accurate Analysis of Small-Diameter Vessels in Cineangiograms. IEEE Trans. Med. Imag. 16(1) (1997)

17. Li, K., Wu, X., Chen, D.Z., Sonka, M.: Efficient Optimal Surface Detection: Theory, Implementation and Experimental Validation. SPIE Medical Imaging (2004)

18. Moon, T.K., Stirling, W.C.: Mathematical Methods and Algorithms for Signal Processing. Prentice Hall, Inc, Englewood Cliffs (2000) 